\title{
Enhancement of Liquid-Solid Two-Phase Flow Through a Vertical Swirling Pipe
}

\author{
J. Yin ${ }^{1}$, Q. Y. Chen ${ }^{2}$, R. Zhu ${ }^{3}$, W. X. Tang ${ }^{1}$, S. J. Su${ }^{1}$, F. Yan ${ }^{1 \dagger}$ and L. H. Wang ${ }^{1}$ \\ ${ }^{1}$ School of Mechanical Engineering, Jiangsu University of Science and Technology, Zhenjiang 212000, \\ China \\ ${ }^{2}$ Department of Metallurgy and Automotive Engineering, Shandong Vocational College of Industry, Zibo \\ 256414, China \\ ${ }^{3}$ School of Environmental and Chemical Engineering, Jiangsu University of Science and Technology, \\ Zhenjiang 212000, China
}

$\dagger$ Corresponding Author Email: yanfei@just.edu.cn

(Received November 20, 2019; accepted March 22, 2020)

\begin{abstract}
In order to improve the transportation efficiency and safety of the vertical hydraulic transport pipe, a new type of pipeline transport system with helical blade is proposed in this paper. Based on CFD-DEM coupling method, the liquid-solid two-phase flow characteristics are analyzed for the swirling pipes and no blade pipe. The study focuses on the effect of the different helix angles of helical blade pipes in terms of the distributions of fluid velocity, the fluid vorticity, the total pressure, the particle's local concentration, the drag force and kinetic energy of particles. Subsequently, the transport efficiency is measured based on the starting speed of particles and the particle concentration, and the safety of the particle transportation is evaluated based on the flow structure and the kinetic energy of particles. It is found that the tangential velocities of the swirling pipes are clearly larger than that of the case of no blade pipe, and the swirling number decreases as the increasing of helix angle of helical blades within swirling pipe. As the decreasing of helix angle, the vorticity magnitude increases sequentially, and the vortex core structure of the flow field is gradually enriched. Meanwhile, the total pressures for the swirling pipes decrease rapidly after the fluid enters the helical blades region, reflecting the difference energy efficiency of the swirling pipes. Furthermore, the swirling pipe accelerates the starting speed of the particle, and then increases the particle concentration in the pipe while making the particle spatial flow structure better and the particle kinetic energy larger. In general, the swirling pipe makes the particle transportation more efficient and safe.
\end{abstract}

Keywords: Liquid-solid two-phase; Kinetic energy; Swirling flow; CFD-DEM; Vorticity.

\section{NOMENCLATURE}

CFD Computational Fluid Dynamics

$C_{v l} \quad$ local concentration

DEM Discrete Element Method

$E_{k t} \quad$ total kinetic energy of particle group

$F_{c} \quad$ collision force

$F_{D} / F_{G}$ dimensionless drag force

$F_{w} \quad$ transfer quantity of liquid-solid phase

$k \quad$ turbulent kinetic energy

$P \quad$ total pressure

$P^{*} \quad$ dimensionless total pressure

$Q \quad Q$-criterion

$S \quad$ swirling number

\section{INTRODUCTION}

With the depletion of terrestrial mineral resources and the development of human understanding of the

$\begin{array}{ll}S_{i j} & \begin{array}{l}\text { antisymmetric tensor in the velocity } \\ \text { gradient tensor }\end{array} \\ v_{a} / U_{l} & \begin{array}{l}\text { dimensionless axial velocity } \\ V_{p a r}\end{array} \\ V_{p i} & \text { volume of particle } \\ v_{f} / U_{l} & \text { volume of the pipe } \\ & \\ \alpha & \text { void ratio } \\ \varepsilon & \text { turbulent dissipation rate } \\ \rho & \text { fluid density } \\ \Omega_{i j} & \text { symmetric tensor in the velocity gradient } \\ & \text { tensor }\end{array}$

deep sea, countries around the world have turned their attentions to the oceans that have not yet been systematically developed. Large-scale exploitation of Marine resources has become an important 
strategic goal of all countries in the world, and deep-sea metal mineral resources will surely become the replacement of resources needed by human beings in the 21st century (Tang et al. 2013 and Xiao et al. 2014). The deep-sea metal mineral resources enriched in the deep ocean belong to the category of coarse particles, and the deep-sea mining technology that collects, breaks and elevates the deep-sea metal mineral resources stored in the seabed thousands of meters deep in the ocean from the ocean bottom to ships is extremely important (Hein 2003 and Baker 2007). According to the existing research results of deep-sea mining technology, vertical pipeline hydraulic lifting system is most likely to become the first generation of the commercial mining system (Tang et al. 2013).

Pipeline hydraulic transportation is a kind of transportation modes that use liquid (usually water) as the carrier to transport solid materials through pipelines. Pipeline transportation technology has been widely applied in the engineering field due to its advantages of no pollution, energy saving, no weather impact and large transportation volume (Bai et al. 2001). At present, the research on hydraulic transport of solid particles in pipelines mainly focuses on the study of solid-liquid twophase flow characteristics. Newitt et al. (1955) proposed four movement patterns of homogeneous flow, heterogeneous flow, bedded flow and fixed sediment flow according to the movement characteristics of particles in the horizontal pipe. Durand (1952) established the relationship of the critical flow rate by taking pipe diameter $\mathrm{D}=0.04 \sim 0.58 \mathrm{~m}$ and particle size $\mathrm{D}=0.44 \sim 2.04 \mathrm{~mm}$ as experimental conditions. $\mathrm{Xu}$ et al. (1998) proposed a new model for the distribution of heterogeneous flow velocity in horizontal pipes from the analysis of momentum transfer of particles and carriers. Cao et al. (2012) studied the motion state and critical condition of particles in horizontal pipe based on different particle sizes, volume concentrations and conveying velocities, and proposed the calculation formula of critical condition. Asakura et al. (1997) adopted numerical simulation method to simulate the hydraulic lifting process of the vertical pipe. The lifting material was glass beads with $2 \mathrm{~mm}$ diameter, and the local average velocity of the liquid phase was obtained. Xia et al. (2004) adopted a new method to calculate the hydraulic gradient in the vertical pipeline, and established a formula to calculate the total hydraulic gradient of the mixture flow under different flow and solid load conditions. Among the current research results on liquid-solid two-phase flow characteristics, there are plenty of studies on horizontal pipe and relatively few studies on vertical pipe. Meanwhile, most of the research objects are fine particle slurry, and the motion state of coarse particles in the pipe is very complicated. Therefore, it is extremely necessary to study the characteristics of solid-liquid two-phase flow during the transportation of coarse particles in vertical pipes.

The traditional deep sea mining hydraulic lifting system mostly adopts axial flow conveying, while the swirling flow has the advantages of concentrated energy, strong carrying capacity and high conveying concentration compared with the axial flow. The structure of the guiding device determines the intensity and structure of the swirling flow, Bali and Sarac (2014) developed a propeller-type guiding device that introduced attenuating vortices and analyzed their effects on pressure drop and heat transfer in the flow field. $\mathrm{Li}$ and Tomita (2000) used a radial vane-type guiding device to conduct an experimental study on the characteristics of particles in horizontal swirling pneumatic conveying, and measured the particle velocity distribution and region concentration using photographic image technology. Escudier et al. (1980) adopted the tangential inlet type guiding device on the end-surface, obtained the LDA measurement results of the finite turbulence vortex, and analyzed the significant change trend of its vortex structure. Derksen (2005) simulated the turbulence in the tangential inlet pipe, analyzed the average velocity distribution of the entire vortex tube, and concluded that it was closely related to the pipe diameter of the outlet. Fokeer et al. (2009, 2010) studied a three-lobed spiral type guiding device, analyzed its axial and tangential velocity distribution and the attenuation law of swirl number, and verified the reliability of the test through numerical simulation. Zhou et al. (2016) combined the experimental device of Fokeer et al. $(2009,2010)$ with rifle pipe and guide vane pipe, and comprehensively analyzed the velocity distribution, pressure drop, particle distribution and energy consumption of the flow field guided by different device. However, most of the abovementioned swirling guiding devices were applied in pneumatic swirling conveying, and most of the materials in conveying are fine particles, so they had limitations of application in the deep-sea mining condition. The propeller-type guiding devices developed by Bali et al. (2014) and Li et al. (2000) have strong swirling intensity, but the material particles could not pass the guiding device, which was not applicable in the deep sea mining conditions. The tangential jet guiding device studied by Escudier et al. (1980) and Derksen (2005) had the highest intensity, but it consumed plenty of energy and was difficult to implement in the deepsea environment. The helical wall devices studied by Fokeer $(2009,2010)$ and Zhou et al. (2016), such as three-lobed spiral pipe and rifle pipe, were relatively reliable and feasible in structure. But the swirling intensity of helical wall devices was relatively low and the processing of pipe was relatively difficult.

In this paper, a new type of pipeline transport system with helical blade is proposed to improve the transportation efficiency and safety of the vertical hydraulic transport pipe. Based on CFDDEM coupling method, the liquid-solid two-phase flow characteristics are analyzed for the swirling pipes and no blade pipe. The study focuses on the effect of the different helix angles of helical blade pipes in terms of the distributions of fluid velocity, the fluid vorticity, the total pressure, the particle's 
local concentration, the drag force and kinetic energy of particles.

\section{MethodOlOGIES}

\subsection{Governing Equation}

Under the Euler-Lagrangian framework, the liquid phase and solid phase models were adopted based on the CFD-DEM coupling method. Since the fluid velocity analyzed in this paper was much lower than the sound speed and there was no significant heat exchange in the process of transport, it was only necessary to define the liquid phase control equation from the aspects of mass conservation and momentum conservation.

\subsubsection{Governing Equation of the liquid phase}

The Navier-Stokes equations are used to solve the continuous liquid-phase flow (Fokeer et al. 2010):

$$
\begin{aligned}
& \frac{\partial \alpha \rho_{l}}{\partial t}+\nabla \cdot \rho_{l} \alpha U_{l}=0 \\
& \frac{\partial \alpha \rho_{l} U_{l}}{\partial t}+\nabla \cdot \rho_{l} \alpha U_{l} \otimes U_{l}=-\nabla \cdot p+\nabla \cdot\left(\mu_{l} \alpha \nabla U_{l}\right)+\rho_{l} \alpha g-F_{w}
\end{aligned}
$$

Where $\rho_{l}$ is the fluid density, $t$ is the time, $U_{l}$ is the fluid velocity, $\mu_{l}$ is the fluid viscosity, $\alpha$ is the void ratio, $g$ is the gravitational acceleration, and $p$ is the pressure. $F_{w}$ is the transfer quantity of liquid-solid phase:

$$
F_{w}=F_{\text {drag }}+F_{\text {Magnus }}+F_{\text {Saffinan }}
$$

The Realizable $k-\varepsilon$ turbulence model (Shih et al. 1995) can well simulate the flow conditions of swirl, shear flow and flow in the pipeline. The governing equation is described by:

$$
\begin{aligned}
& \frac{\partial}{\partial t}\left(\rho_{l} k\right)+\frac{\partial}{\partial x_{i}}\left(\rho_{l} k u_{l}\right)=\frac{\partial}{\partial x_{j}}\left(\left(\mu_{l}+\frac{\mu_{t}}{\sigma_{k}}\right) \frac{\partial k}{\partial x_{j}}\right)+G_{k}-\rho_{l} \varepsilon \\
& \frac{\partial}{\partial t}\left(\rho_{l} \varepsilon\right)+\frac{\partial}{\partial x_{i}}\left(\rho_{l} \varepsilon u_{l}\right)=\frac{\partial}{\partial x_{j}}\left(\left(\mu+\frac{\mu_{t}}{\sigma_{\varepsilon}}\right) \frac{\partial \varepsilon}{\partial x_{j}}\right)+\rho_{l} C_{1} E \varepsilon-\rho_{l} C_{2} \frac{\varepsilon^{2}}{k+\sqrt{v_{l} \varepsilon}}
\end{aligned}
$$

Where $k$ and $\varepsilon$ represent the turbulent kinetic energy and the turbulent dissipation rate, respectively. $\sigma_{k}$, $\sigma_{\varepsilon}, C 1$ and $C 2$ are 1, 1.2, 1.44 and 1.9, respectively; $\sigma_{k}, \sigma_{\varepsilon}$ are the Prandtl numbers corresponding to the turbulent energy $k$ and the dissipation rate $\varepsilon, C_{l}$ and $C_{2}$ are constants. $v_{l}$ is the kinematic viscosity coefficient, and $u l$ is the mean velocity of liquid (Shih et al. 1995 and Ghaya et al. 2019).

\subsubsection{Governing Equation of The Particle Phase}

The Newton's law of motion is used to describe the particle's translational and rotational motions (Zhou, 2014):

$m_{p} \frac{d U_{p}}{d t}=F_{\text {drag }}+F_{\text {Saffinan }}+F_{\text {Magnus }}+F_{c}+m_{p} g$

$I_{P} \frac{d \omega_{p}}{d t}=T_{p}$
Where $m_{p}$ and $I_{p}$ are the mass and moment of inertia of the particle, $U_{p}$ and $\omega_{p}$ are the translational velocity and rotational velocity of the particle, $T_{p}$ is the particle moment, $F_{c}$ is the collision force of particle-particle and particle-wall, modeled by the Soft-sphere Contact Model.

Drag force is an important parameter affecting the liquid-solid two-phase momentum exchange. The Ergun (1952) and Wen and Yu (1955) drag model is used to calculate the drag force of each single particle in the flow field:

$F_{d}=\frac{\beta V_{p}\left|U_{l p}\right| U_{l p}}{1-\alpha}$

Where $V_{p}$ is the single particle volume, $U_{l p}$ is the relative motion velocity between water and particles, $U_{l p}=U_{l}-U_{p}, \beta$ is the liquid-solid phase resistance coefficient, which is calculated by

$\beta= \begin{cases}\frac{150(1-\alpha)^{2} U_{l l}}{e d_{e q}^{2}}+\frac{1.75(1-\alpha) \rho_{l}\left|U_{l p}\right|}{d_{e q}} & \varepsilon<0.8 \\ \frac{3}{4} C_{d} \rho_{l} \varepsilon^{-1.65}(1-\alpha)\left|U_{l p}\right| & \varepsilon \geq 0.8\end{cases}$

Where $d_{e q}$ is the equivalent spherical diameter of the particles, which is calculated as spherical particle diameter in this paper; $C_{d}$ is the drag coefficient of single particle.

$C_{d}= \begin{cases}\frac{24\left(1.0+0.25 R e_{p}^{0.687}\right)}{R e_{p}} & R e \leq 1000 \\ 0.44 & R e>1000\end{cases}$

After introducing the void ratio $\alpha$, the particle Reynolds number $R_{e p}$ is expressed as

$R e_{p}=\frac{\alpha \rho_{l} d_{e q}\left|U_{l p}\right|}{\mu_{a}}$

The Magnus force and Saffman force are small when compared with the drag force, and Tsuji et al. (2008), Karimi and Dehkordi (2015) have made a detailed explanation.

\subsection{Simulation Modeling}

The pipe model analyzed in this study contains the helical blades with a certain helix angle. The pipes with the helix angles of $30^{\circ}, 45^{\circ}$ and $60^{\circ}$ were selected as the research objects, and the diameter of the pipe was $200 \mathrm{~mm}$. Figure 1 showed the schematic diagram for geometry of three types blades, and different pipelines were abbreviated by working condition codes BL1, BL2, BL3 and NBL respectively, as shown in Tab. 1. And BL1, BL2, BL3 are collectively referred to as swirling pipes.

Tab 1 Working condition codes for all cases

\begin{tabular}{|c|c|}
\hline Working condition & Code \\
\hline $\begin{array}{c}\text { Helical blade with } 30^{\circ} \text { helix angle } \\
\text { inside the pipe }\end{array}$ & BL1 \\
\hline $\begin{array}{c}\text { Helical blade with } 45^{\circ} \text { helix angle } \\
\text { inside the pipe }\end{array}$ & BL2 \\
\hline $\begin{array}{c}\text { Helical blade with } 60^{\circ} \text { helix angle } \\
\text { inside the pipe }\end{array}$ & BL3 \\
\hline No blade pipe & NBL \\
\hline
\end{tabular}



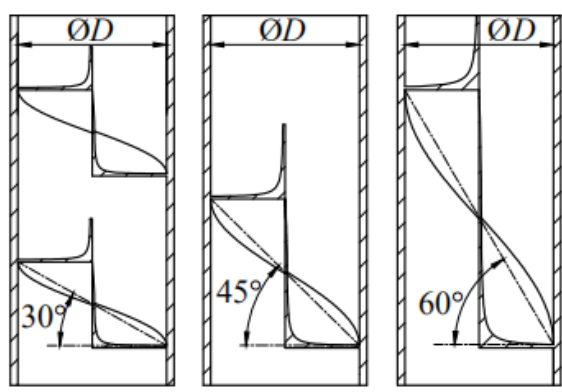

Fig. 1. Schematic diagram for the helical blade pipes with three different helix angles.

Figure 2 showed the schematic diagram of the conveying process of swirling pipe. Considering the existence of the helical blade, the numerical calculation model was divided into three calculation areas: inlet region, helical blade region and downstream region.

The grids of different computational domains were divided separately. The inlet region and the downstream region were divided by a hexahedral structured grid. The helical blade region was divided by a tetrahedral unstructured grid, and both structured and unstructured grid segments were refined near boundary, including grids near the blade surface. The interface face was used to connect incompatible computing domains, as shown in Fig. 3.

During the CFD-DEM coupling process, Fluent first calculates the flow field at a certain time point until the convergence of residual, and uses the drag force model to convert the information of flow field into the drag force acting on the particles in EDEM. Subsequently, EDEM calculates the external force (drag force, gravity, collision force, etc.) on each particle, and updates the position and velocity of the particles. Finally, the properties of these particles are added to the CFD calculation in the form of momentum sink, which affects the flow field, and a new round of calculation is subsequently performed.

In the CFD calculation, the pressure-based solver was used due to the fluid velocity was much lower than the speed of sound, and the Realizable k- $\varepsilon$ turbulence model was selected. The Standard wall function was used to calculate the near-wall flow, which can ensure the accuracy of the calculation results and keep the number of grids on a reasonable order of magnitude (Ariyaratne et al. 2007). The governing equations were discretized based on the finite volume method, and the QUICK discretization scheme was used to solve the momentum and turbulence equations. The SIMPLE algorithm was applied to ensure pressure-velocity coupling. Further, in the boundary condition, the inlet of the flow field was set as the velocity inlet, the outlet was set as the pressure outlet $(0 \mathrm{~Pa})$, and the rest were no-slip walls.

In the CFD-DEM coupling calculation, the Eulerian-Lagrangian method was adopted for coupling control, and the Ergun (1952) and Wen and Yu (1955) drag model was selected, and Saffman lift and Magnus lift were considered at the same time. The remaining simulation parameters applied in this work were shown in Tab. 2 .

\subsection{Model Validation}

\subsubsection{Tests of Grid Size Independence}

In order to verify the accuracy of the model, the numerical model was divided by four different grid sizes, and four different time steps were used in the CFD numerical calculation. In CFD-DEM calculation, the CFD time step was generally between 10 and 100 times the DEM time step, so the CFD calculation time step was set to $0.01 \mathrm{~s}$, $0.007 \mathrm{~s}, 0.004 \mathrm{~s}, 0.001 \mathrm{~s}$, respectively. The total simulation time of all cases was set to $10 \mathrm{~s}$, the maximum number of iterations per time step was 30 and the convergence residual standard was $1 \mathrm{E}-5$.

In this paper, the Standard wall function was adopted to calculate the flow of the near-wall region, so the node of the first-layer grid was arranged in the log-Law layer, that is, the range of $y+$ is $30-300$ (Xiong et al. 2012). The height of the first-layer grid was estimated by Eq. 12 (Schlichting 1979), and then the numerical calculation is carried out to check the actual value of $y+$.

$y^{+}=0.172 \frac{\Delta y}{L} \operatorname{Re}_{L}^{0.9}$

Where $\Delta y$ is the height of the first-layer grid, $L$ is the reference length, $R e$ is the Reynolds number.

Hence, when meshing, the $y+$ was taken as 40 . The near-wall region of the grid was divided based on the first-layer grid height of $0.0003 \mathrm{~m}$ and the grid increasing ratio of 1.2. Tab. 3 showed the parameters of the four different grid sizes.

Table 3 Grid parameters

\begin{tabular}{|c|c|c|}
\hline Grid & $\begin{array}{c}\text { Number of } \\
\text { nodes }\end{array}$ & $\begin{array}{c}\text { Number of } \\
\text { cells }\end{array}$ \\
\hline Coarse grid & 112084 & 212966 \\
\hline Medium grid C & 208046 & 469557 \\
\hline Medium grid F & 451290 & 916966 \\
\hline Fine grid & 672058 & 1591154 \\
\hline
\end{tabular}

The cross section (14D) twice the pipe diameter from the outlet of the generator section was selected as the observation plane for comparison, and the maximum axial velocity was selected as the analytical index, as shown in Fig. 4

As shown in Fig. 4, the maximum axial velocity at the 14D observation plane varied with the change of the grid size and the time step, and the maximum axial velocity was approximately positively correlated with the calculation time step. But when the mesh size was reduced to the Medium grid $\mathrm{F}$ and time step was reduced to $0.004 \mathrm{~s}$, the trend was no longer obvious. Hence, the CFD calculation time step was set to $0.004 \mathrm{~s}$, and the Medium mesh F with a grid number of 916966 was selected in the numerical simulation. 

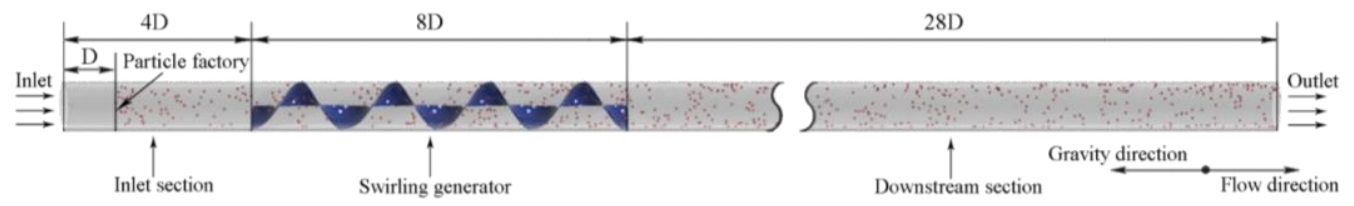

Fig. 2. Schematic diagram of the conveying process of swirling pipe.

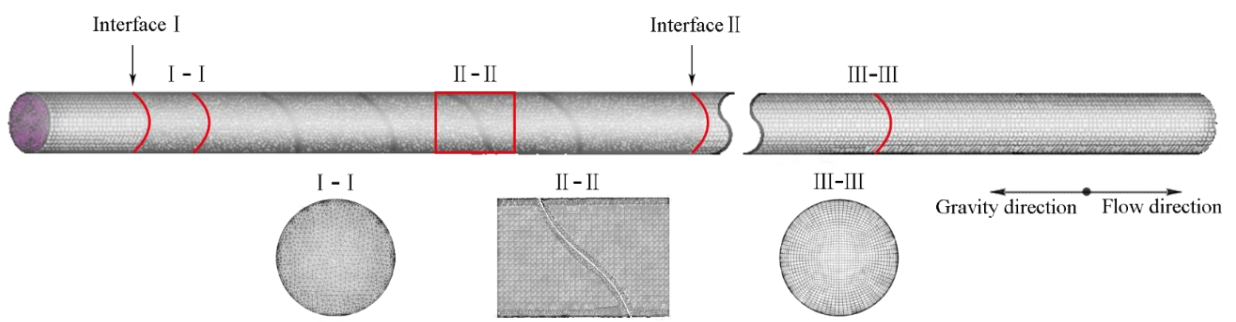

Fig. 3. Fluid domain meshing of swirling pipe.

Table 2Parameters of simulation

\begin{tabular}{|c|c|c|c|c|c|}
\hline Phase & Item & Details & Index & Unit & Value \\
\hline \multirow[t]{7}{*}{ CFD } & Materials & Fluid/water & Density & $\mathrm{kg} \cdot \mathrm{m}^{-3}$ & 1025 \\
\hline & & & Viscosity & $\mathrm{kg} \cdot \mathrm{m}^{-1} \cdot \mathrm{s}^{-1}$ & $1.003 \mathrm{E}-3$ \\
\hline & $\begin{array}{l}\text { Boundary } \\
\text { conditions }\end{array}$ & Velocity-inlet & Velocity magnitude & $\mathrm{m} \cdot \mathrm{s}^{-1}$ & 3 \\
\hline & & Turbulence & Turbulence intensity & - & $3 \%$ \\
\hline & & Pressure outlet & Gauge pressure & $\mathrm{Pa}$ & 0 \\
\hline & & Wall & Wall motion & & Stationary wall \\
\hline & & & Shear condition & & No slip \\
\hline \multirow[t]{19}{*}{ DEM } & Materials & Particle & Poisson's ratio & - & 0.25 \\
\hline & & & Shear modulus & $\mathrm{Pa}$ & $1 \mathrm{E}+8$ \\
\hline & & & Density & $\mathrm{kg} \cdot \mathrm{m}^{-3}$ & 2040 \\
\hline & & Wall & Poisson's ratio & - & 0.3 \\
\hline & & & Shear modulus & $\mathrm{Pa}$ & $7 \mathrm{E}+10$ \\
\hline & & & Density & $\mathrm{kg} \cdot \mathrm{m}^{-3}$ & 7800 \\
\hline & Interaction & Particle-particle & Coefficient of restitution & - & 0.525 \\
\hline & & & Coefficient of static friction & - & 0.642 \\
\hline & & & Coefficient of rolling friction & - & 0.05 \\
\hline & & & Interaction contact model & & Hertz-Mindlin(no slip) \\
\hline & & & Collision model & & Soft-sphere Contact Model \\
\hline & & Particle-wall & Coefficient of restitution & - & 0.525 \\
\hline & & & Coefficient of static friction & - & 0.4 \\
\hline & & & Coefficient of rolling friction & - & 0.05 \\
\hline & & & Interaction contact model & & Hertz-Mindlin(no slip) \\
\hline & & & Collision model & & Soft-sphere Contact Model \\
\hline & Particle & Particle factory & Particle radius & $\mathrm{mm}$ & 5,10 \\
\hline & & & Factory type & & Dynamic/unlimited number \\
\hline & & & Target mass & $\mathrm{kg} / \mathrm{s}$ & 7.5 \\
\hline
\end{tabular}

\subsubsection{Comparison of The Experimental} Data

Gillies and Shook (1994) studied the concentration distributions for sand slurries flowing in horizontal pipes. The diameter of pipes used in the experiment ranged between 50 and $500 \mathrm{~mm}$, and the particle diameters ranged between 0.18 and $2.4 \mathrm{~mm}$. Among a large number of experimental data measured by Gillies, the experimental condition of a flow velocity of $3.9 \mathrm{~m} / \mathrm{s}$ and a pipe diameter of $0.263 \mathrm{~m}$ was selected for numerical simulation. The Reynolds number of this experimental condition was $9 \mathrm{E}+10$, which was on the same order of 
magnitude as the simulation conditions studied in this paper. In this condition, the particle size is $0.55 \mathrm{~mm}$ and the particle concentration is $15 \%, 25 \%$ and $30 \%$, respectively.

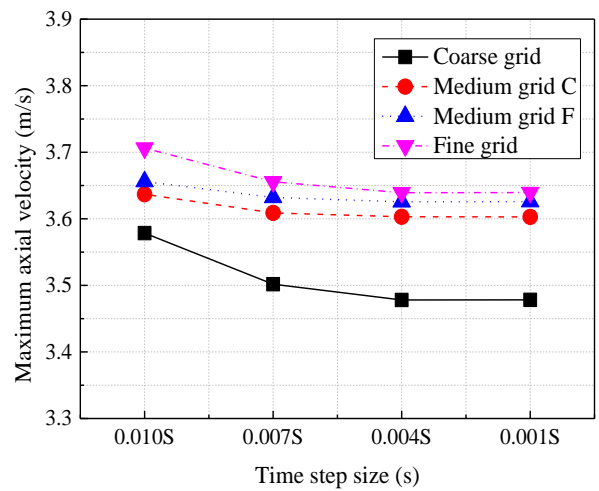

Fig. 4. Maximum axial velocity at 14D position for different Grid sizes and time steps.

In this comparison, the Realizable $\mathrm{k}-\varepsilon$ turbulence model and the SIMPLE algorithm and the QUICK discretization scheme were adopted in Fluent, and the Standard wall function was adopted to calculate the near-wall region. In EDEM, the particles were set as spherical particles to reduce the cost of calculation, and the particle density was $2650 \mathrm{~kg} / \mathrm{m} 3$. The Hertz-Mindlin (no slip) model was selected to control the particle-wall and particleparticle contact. After the numerical simulation, the results were compared with Gillies's experimental data, as shown in Fig. 5.

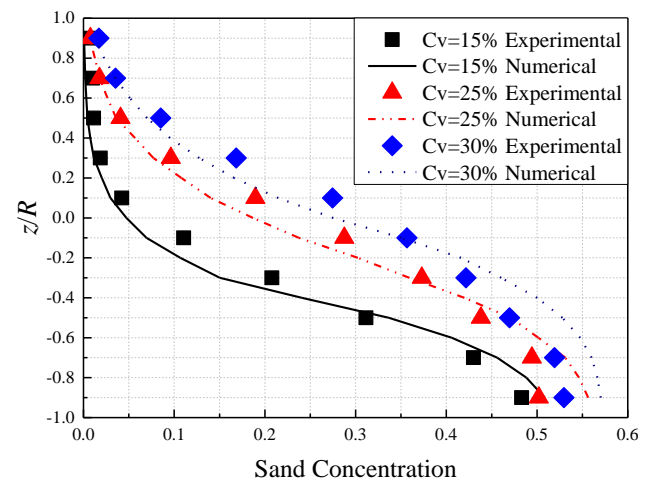

Fig. 5. Comparison of the concentration distribution for different conditions.

In Fig. 5, because of the full development of the flow at 50D section, the particle concentration distributions of experimental data and numerical simulation data were compared at 50D section. As shown is Fig. 5, the simulated data agreed well with the experimental data of Gillies, especially in low concentrations $(15 \%)$ conditions. The error existed because of the different operating modes of simulation and experiment. For example, Gillies added particles to the tank for mixing in the experiment, which has some errors with the particle concentration generated in the particle factory in
EDEM. In addition, Gillies noted that the overpumping flow of particles resulted in the formation of a small amount of powder which affected the concentration distribution.

It was also shown in Fig. 5 that in the upper part of the pipe, the simulated data was in great agreement with the experimental data, while there was some acceptable error at the bottom near- wall region. However, the working condition studied in this paper was vertical pipe transportation, and there would not be a large number of particles stacked on the wall. Therefore, the model selected in this paper was very reasonable, and the simulation results were highly reliable.

\section{RESUlts AND DiscuSSIONS}

\subsection{Fluid Flow}

The flow characteristics analysis of the fluid phase for the swirling hydraulic transport is the basis for studying the multiphase flow characteristic, and then the flow velocity distribution, swirling intensity and vortex structure are analyzed to clarify the flow field characteristics in the swirling pipes and no blade pipe.

\subsubsection{Flow Velocity Distribution}

Figure 6 shows the comparison of the dimensionless axial velocity $\left(v_{a} / U_{l}\right)$ and tangential velocity $\left(v_{t} / U_{l}\right)$ of the three different swirling pipes with no blade pipe at different observation planes along the conveying direction $(y), U_{l}$ is the fluid velocity of the inlet. The average value of axial and tangential velocity of each observation plane are counted during a cycle of monitored pressure fluctuations, The horizontal axis is the dimensionless radial distance of the pipe $(r / R)$, and the flow velocities are analyzed at four section locations: $y=14 \mathrm{D}, y=$ $16 \mathrm{D}, y=18 \mathrm{D}$, and $y=20 \mathrm{D}$ from the pipe inlet.

As shown in Fig. 6(a), the distribution characteristics of $v_{a} / U_{l}$ in the swirling pipes are significantly different from no blade pipe at different observation planes. At $y=14 \mathrm{D}$, a symmetrical warhead-like distribution structure is presented in the no blade pipe, while swirling pipes have a distinct asymmetric distribution. Although the $v_{a} / U_{l}$ of BL2 and BL3 exhibits an asymmetric distribution, it approximates the distribution structure of NBL, while BL1 exhibits an M-shaped axial velocity distribution. Meanwhile, from BL1 to BL3, the maximum axial velocity decreases in turn, but both of them are higher than the maximum axial velocity of the NBL.

From $16 \mathrm{D}$ to $20 \mathrm{D}$, the axial velocity distributions of all cases are similar to the location of 14D section. As the observation plane is farther away from the exit of the helical blade region, the peaks of the axial velocity decrease in BL1, BL2, BL3, but the opposite trend is shown in the NBL. The axial velocity of swirling pipes tends to be symmetrically distributed and the maximum axial velocity of BL2 exceeds that of BL1.

The fluid velocity in the pipe is divided into axial 
J. Yin et al. / JAFM, Vol. 13, No. 5, pp. 1501-1513, 2020.
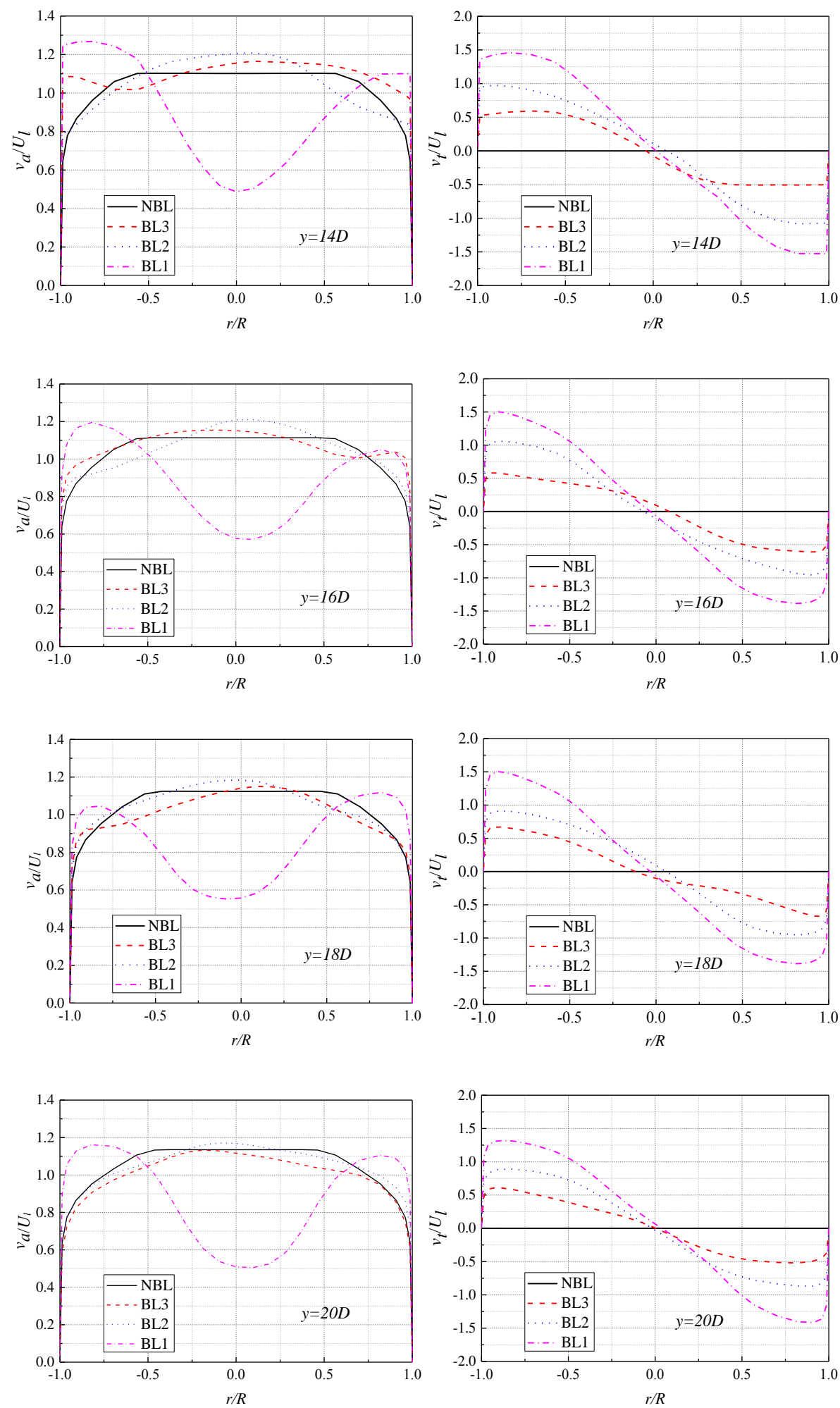

(a) Dimensionless axial velocity $\left(v_{a} / U_{i}\right)$

(b) Dimensionless tangential velocity $\left(v_{t} / U_{i}\right)$

Fig. 6. Profiles of fluid velocity distributions for the three different swirling pipes and no blade pipe.

Table 4 Equations of swirling number for three helical blade pipes

\begin{tabular}{|c|c|c|}
\hline BL1 & BL2 & BL3 \\
\hline$S=0.97178 e^{-0.01835 / / d}$ & $S=0.57803 e^{-0.01748 / / d}$ & $S=0.34395 e^{-0.01493 / / d}$ \\
\hline
\end{tabular}


velocity and tangential velocity, and the magnitude of the tangential velocity directly affects the swirling intensity. As is shown in the $y=14 \mathrm{D}$ section in Fig. 6(b), the dimensionless tangential velocity $\left(v_{t} / U_{l}\right)$ has a significant difference in magnitude from BL1 to BL3. The $v_{t} / U_{l}$ of BL1 is the highest, BL2 is the second, and BL3 is the smallest, while $v_{t} / U_{l}$ of the NBL is too weak to be ignored. The $v_{t} / U_{l}$ of BL1 is about 1.5 times that of BL2, which is more than twice that of BL3. This indicates that the swirling intensity of BL1 should be significantly stronger than others.

From the $16 \mathrm{D}$ to $20 \mathrm{D}$ section, the distribution of $v_{t} / U_{l}$ all cases are same as the $14 \mathrm{D}$ section. The difference is that along the flow direction, $v_{t} / U_{l}$ gradually decreases at each section, representing the gradual decay of the swirling intensity.

\subsubsection{Swirling Intensity}

The swirling number, the most common dimensionless index, is used to characterize the swirling intensity to quantify the swirling characteristics represented by the tangential velocity in the swirling pipes field.

The physical meaning of the swirling number is the ratio of the hoop flux to the axial flux in the flow field (Fokeer et al. 2009, Klančišar et al. 2016, Li and Tomita, 1994, Li and Tomita, 1996 and Algifri et al. 1987), which is defined by

$S=\frac{1}{R} \times \frac{\int_{0}^{R} v_{A} v_{t} r^{2} d r}{\int_{0}^{R} v_{A}^{2} r d r}$

Where $v_{\mathrm{A}}$ and $v_{\mathrm{t}}$ are the axial and tangential velocities, respectively; $R$ is the pipe radius; $r$ is the radial distance.

After the calculation by using Eq. (13), the swirling number (S) is presented in Fig. 7, and the tangential velocity of the NBL is too small to be ignored. Figure 7 shows that the swirling number gradually decreases along the flow direction, and the swirling number of BL1 is the largest, which is about 1.7 times and 2.6 times that of BL2 and BL3, respectively. However, the decay of the swirling number of BL1 is also the fastest. From 16D to $36 \mathrm{D}$, the decay rate of the swirling number of BL1 is about $32 \%$, and the decay rates of the swirling number of BL2 and BL3 are $29 \%$ and $24 \%$, respectively.

As was strongly validated by $\mathrm{Li}$ and Tomita (1994) and Algifri et al. (1987), the decay of swirling number was also accorded with the following exponential law

$$
S=\mathrm{S}_{0} e^{c l / d}
$$

Where $\mathrm{S}$ and $\mathrm{C}$ are constants, and according to Eq. 13 , the residual of each constant is in the order of 1E-5, as shown in Tab. 4.

\subsubsection{Vortex Structure}

The vorticity is defined as the curl of the fluid velocity and is usually used to measure the intensity and direction of the vortex. In this study, the vorticity nephogram of the flow field is drawn on the axis plane in each swirling pipe to visually analyze the swirling intensity, as shown in Fig. 8. In order to properly arrange these images, the images of pipes are placed horizontally.

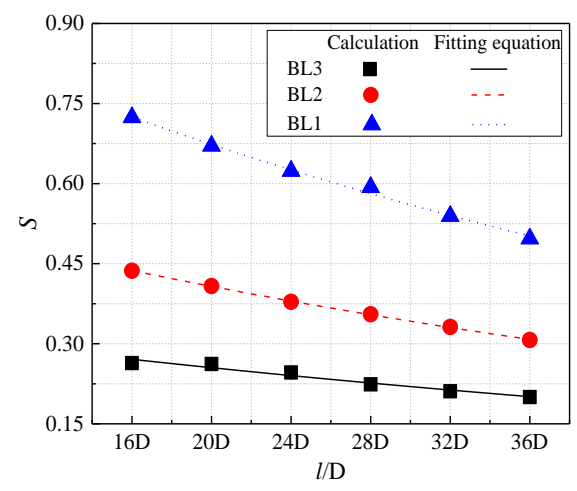

Fig. 7. Swirling number for three different swirling pipes.

The vorticity distributions in all cases are generally similar, as shown in Fig. 8. The vorticity of the inlet region in front of the blade is low, and the maximum vorticity appears in the helical blade region, and then gradually decreases along the flow direction. Moreover, the vorticity value of the central flow region is significantly larger than the near-wall region.

It can be seen that the radial dimension of the vortex sheet in the flow field increases from BL3 to BL1 in sequence. The reason is that the swirling intensity increases in turn due to the increasing of fluid tangential velocity from BL3 to BL1 (Fig.6. b). Therefore, a larger low-pressure zone is needed to balance the centrifugal force. This implies the radial dimension of the central flow region becomes larger as the swirling intensity increases.

The fluid velocity gradually increases away from the axis of the pipe in the radial direction, as shown in Fig. 6, which indicates that the helical blade region has a forced vortex region (Prabhansu 2017), that is, the fluid vorticity is large in this region, and it should be strong rotational motion and shearing motion here. Since the vorticity can only shows the magnitude of the vortex, and in order to further analyze the shape and position of the vortex, the $Q$ criterion is introduced to extract the structure of the vortex core. The $Q$-criterion is the quadratic invariants of the velocity gradient tensor. The definition is

$Q=\left(\Omega_{i j} \Omega_{i j}-S_{i j} S_{i j}\right) / 2$

Where $\Omega_{i j}$ and $S_{i j}$ are the symmetric tensor and the antisymmetric tensor in the velocity gradient tensor, respectively. These two tensors definitions are defined as follows:

$S_{i j}=\left(u_{i j}+u_{j i}\right) / 2$ 

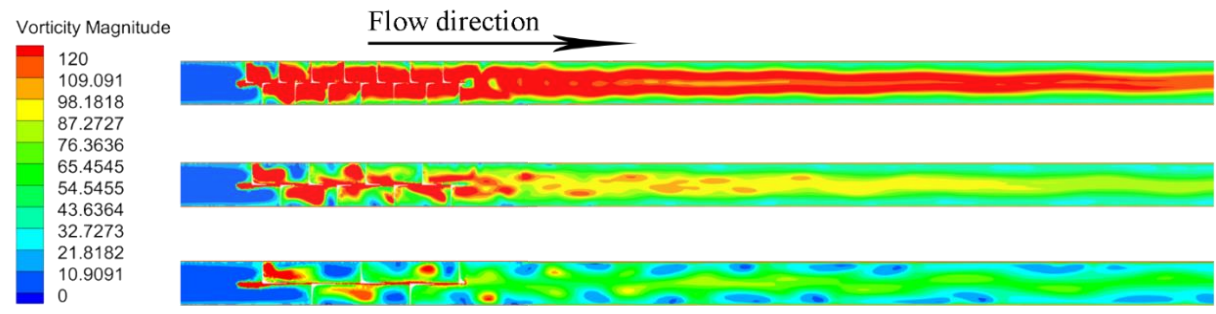

Fig. 8. Vorticity magnitude nephogram of the flow field for three different swirling pipes.

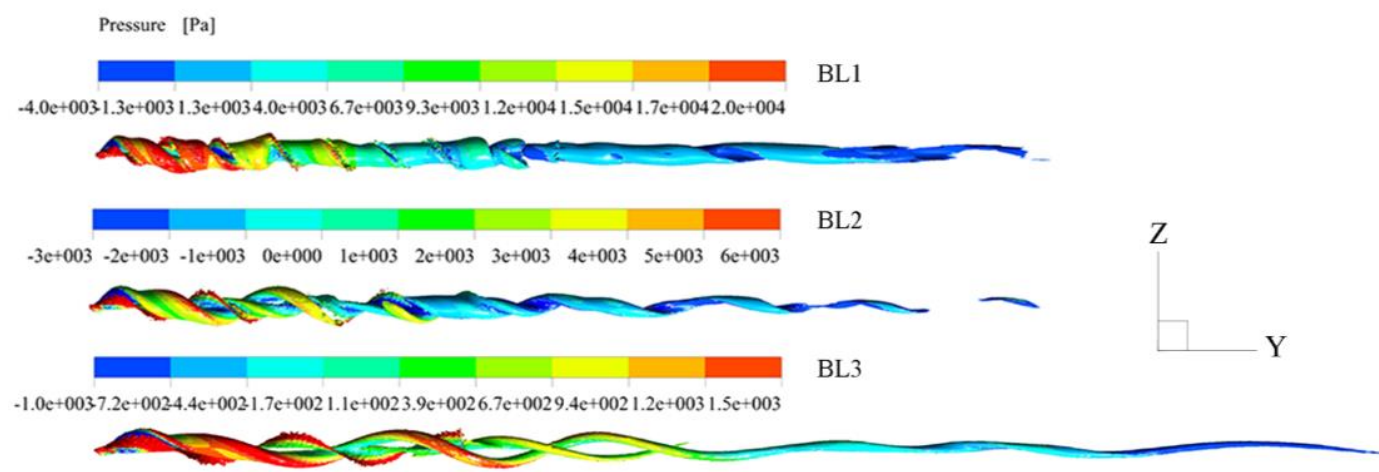

Fig. 9. Pressure distribution of three different swirling pipes based on equal vorticity surface.

$\Omega_{i j}=\left(u_{i j}-u_{j i}\right) / 2$

$Q$ indicates the extent to which $\Omega_{i j} \Omega_{i j}$ is larger than $S_{i j} S_{i j}$. The $Q$-criterion is the response to the balance between the deformation and rotation of the fluid micelles in the flow field. At the position of $Q>0$, the rotation rate $\Omega$ dominates, that is, the vortex structure dominates in this region.

The BL1 to BL3 vortex structure is extracted with same $Q$ value, and a pressure nephogram is attached to the equal vorticity surface, as shown in Fig. 9.

There is a distinct vortex core in the central region, as shown in Fig. 9, indicating a significant rotational motion of the fluid in this region. From BL3 to BL1, the vortex structure of the flow field is gradually enriched, which is also consistent with the increasing trend of the vorticity. In the flow direction, the vortex structure gradually develops regularly, and at the same time, it gradually breaks down and weakens as the swirling intensity decreases. It can be found that the vortex core of the BL3 which is extracted by the same $Q$ value is the longest, that is, the rupture attenuation rate of the vortex structure of the BL3 is the slowest, which is also consistent with the conclusion that the attenuation rate of the swirl number of BL3 is the smallest (Fig. 7).

\subsection{Load Particles}

\subsubsection{Total Pressure Drop}

The total pressure of the flow field characterizes the total energy of the flow system, and the total pressure drop represents the energy consumption of the system. In order to more clearly analyze the total pressure drop of each working condition, the total pressure of the flow field adopts a dimensionless form:

$$
P^{*}=\frac{P}{3 \rho_{l} u_{l}^{2}}
$$

Where $P^{*}$ is the dimensionless total pressure; $P$ is the total pressure of system, which is a average value counted during a cycle of monitored pressure fluctuations.

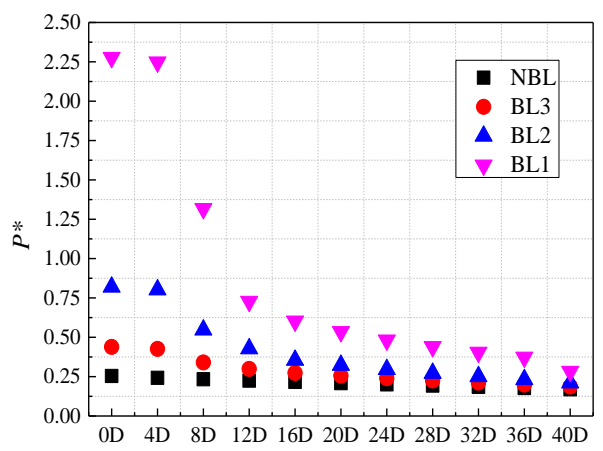

$l / \mathrm{D}$

Fig. 10. The dimensionless total pressure for three different swirling pipes and no blade pipe.

Figure 10 shows the profiles of dimensionless total pressure $P^{*}$ for the three different swirling pipes and no blade pipe. It can be clearly seen that the value of $P^{*}$ decreases in all cases along the flow direction, and eventually they're going to be equal at the outlet of all cases. However, $P^{*}$ for the different helical blades decreased rapidly after the mixed 


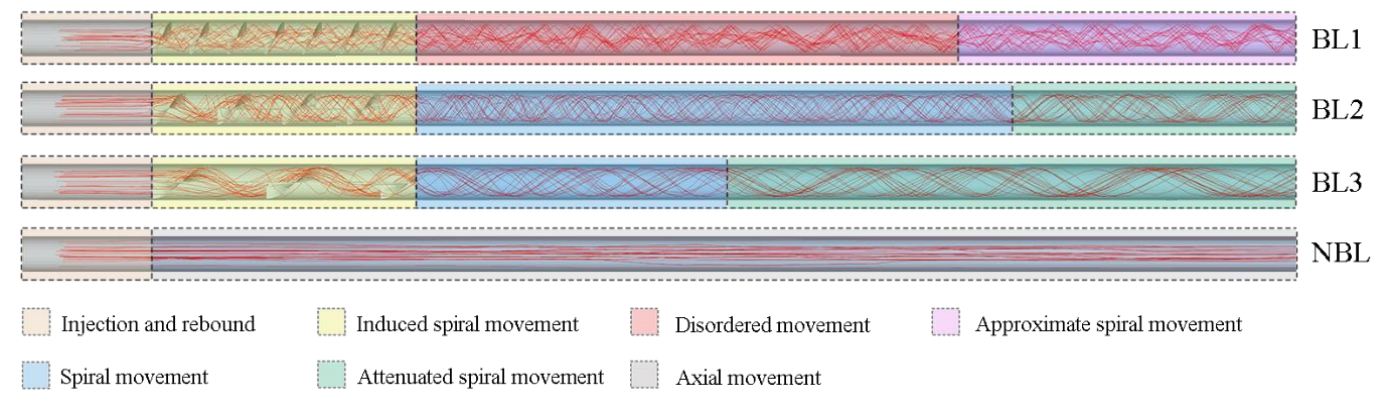

Fig. 11. Particle motion traces for the three different swirling pipes and no blade pipe.

fluid enters the helical blade region $(4 \mathrm{D} \leqslant y \leqslant 12 \mathrm{D})$, reflecting the difference in energy efficiency of different helical blades, and this phenomenon is particularly noticeable in BL1 and relatively flat in BL2 and BL3.

On the other hand, $P^{*}$ of each case has a similar gradient in the downstream region after passing the helical blades, and the curve of the swirling pipe has a decreasing slope which is larger than that of the no blade pipe, this phenomenon indicates that the swirling intensity guided by the helical blade has a certain correlation with the energy loss of the flow field. BL1 has the strongest swirling intensity, but its energy consumption is highest, and BL3 is the opposite. Meanwhile, BL2 can not only produce a strong swirling intensity, but also its energy consumption is relatively small, indicating that BL2 has higher energy efficiency.

\subsubsection{Particle Transport Status}

In order to analyze the motion traces of the particles in different swirling pipes and no blade pipe, 20 particles were randomly selected and their motion traces were extracted, as shown in Fig. 11.

As is vividly shown in Fig. 11 that the particles is concentrated in the center of the pipe in the NBL, and its trace approximates a straight line. Meanwhile, the particles in swirling pipes are gathered near the pipe wall and their motion is a regular quasi-periodic spiral ascending motion, avoiding extreme conditions such as blockage which may occur due to the increase of particle concentration in the center of the pipeline. In the flow direction, the axial movement distance increases in the unit rotation period of the particle, so the ratio of the tangential movement distance of the particle to the axial movement distance gradually decreases, and this is the macroscopic expression of the attenuation of the swirl number.

Comparing the three swirling conditions, it can be found that BL1 has a very strong swirling intensity, which causes the flow state to be disordered. Therefore, the particles just passing through the blade region appear an approximate disordered state, which also causes the pressure drop of BL1 to be higher than that of others cases, and also is the reason why the maximum axial velocity of BL1 decreases and the maximum axial velocity of BL2 becomes the largest (Fig. 6). Subsequently, the particles exhibit a quasi-helical motion state as the swirling intensity decreases. The motions of BL2 and BL3 are composed of spiral motion and attenuating spiral motion, but the spiral motion region of BL2 is longer. As the law of swirl number attenuation shown in Fig. 7, BL3 has the smallest swirling intensity, so the spiral motion region of the particles is shorter than BL2

In order to analyze the influence of particle traces on the local concentration $\left(C_{v l}\right)$, which is defined by

$$
C_{v l}=\frac{V_{p a r}}{V_{p i}}
$$

Where $V_{p a r}$ is the volume of particle in a certain amount of time; $V_{p i}$ is the volume of the pipe in a certain amount of time.

The volume concentration of the particle group was statistically analyzed within $1 \mathrm{~s}$ of different regions of the pipeline, as shown in Fig. 12.

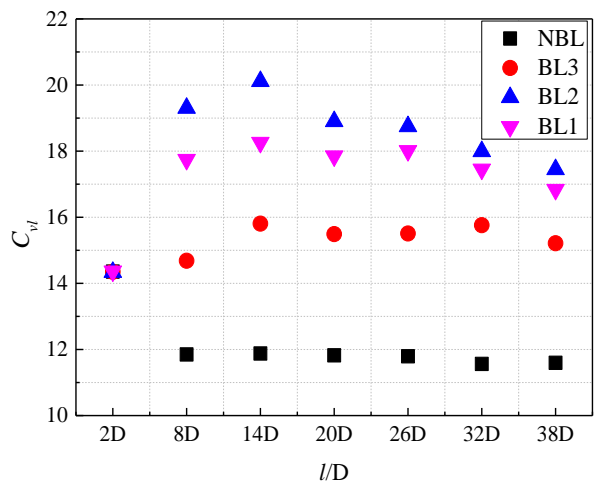

Fig. 12. Local concentration at different observation planes for three different swirling pipes and no blade pipe.

It can be seen that particles are enriched due to the low particle velocity near the inlet of the pipe $(y=$ 2D), and the particle local concentration is slightly larger than the set lifting concentration of numerical calculation, but the local concentration of all cases is basically same. Meanwhile, along the flow direction, the local concentration decreases at different observation planes in no blade pipe and tends to stabilize, while the local concentration first 
increases and then decreases in the swirling pipes.

The local concentration of BL1 and BL2 is between $17 \%$ and $20 \%$, and the local concentration of BL3 is about $15 \%$ 16\%, which is significantly higher than the local concentration of the NBL, forming a transport state of denser phase. This is also the reason why the pressure drop of swirling pipe is larger than that of the no blade pipe in the downstream region after the helical blade (Fig. 10). It's obvious the efficiency of the system is higher for the higher transport concentration, however, it will lead to the aggregation of particles becomes serious. According to the analysis of the particle traces, the particles in the swirling pipes gather spirally at the near-wall surface, and the possibility of blocking is low. The case of BL2 has the highest local concentration and relatively reasonable energy consumption, which indicates that BL2 has the best transport efficiency.

\subsubsection{Drag Force On Particles and Kinetic energy}

The drag force on the particles in hydraulic transport determines the flow structure of particles. Hence, the values of the drag force of the particle group in the statistical domain at different observation sections are averaged over time, and the average value of drag force $\left(F_{D}\right)$ of the particle group is processed by gravity $\left(F_{D} / F_{G}\right)$ to be dimensionless, as shown in Fig.13.

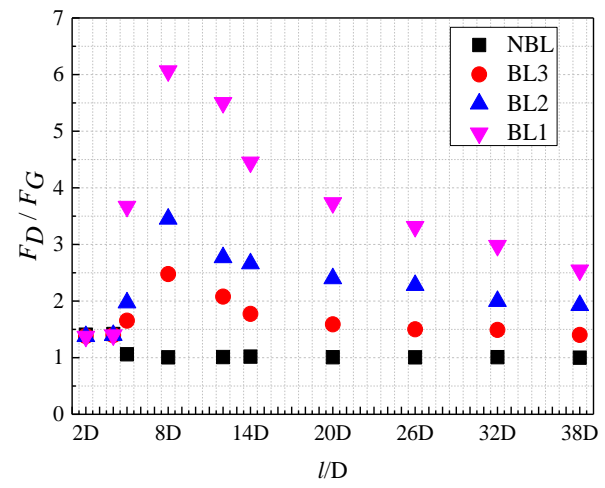

Fig. 13. Dimensionless drag force at different observation planes for three different swirling pipes and no blade pipe.

At the inlet region $(2 \mathrm{D}<y \leqslant 4 \mathrm{D}), F_{D} / F_{G}$ is almost the same in all cases, and then $F_{D} / F_{G}$ varies significantly along the flow direction, as shown in Fig. $13 . F_{D} / F_{G}$ in no blade pipe gradually decreases and tends to be stable along the flow direction, while $F_{D} / F_{G}$ in the swirling pipes first increases and then decreases, and gradually stabilized. $F_{D} / F_{G}$ for the cases of the swirling pipes clearly larger that of no blade pipe in the entire observation area $(2 \mathrm{D}<y$ $\leqslant 38 \mathrm{D}$ ), especially in the helical blade region (4D $<y \leqslant 12 \mathrm{D}$ ). This is because the fluid vorticity is large in this region (Fig. 8), so the cases of swirling pipes formed by the helical blades effectively carry the particles, and the water sufficiently acts on the particles, so that the drag force of particle group is significantly increased. The larger drag force of the swirling pipes allows particles to achieve greater acceleration and faster start-up, improving the safety and efficiency of the most critical start-up period in the transport process.

In the flow direction, the swirling intensity is gradually weakened, and the drag force of the particle group is reduced as well, but still larger than the drag force of the particle group in the no blade pipe. As the particles enter the downstream region, the flow environment of particle in no blade pipe and the swirling pipes is similar, so the drag force of the particle group has a similar change trend in each case.

The enough kinetic energy of the particle is one of the important reasons to ensure the safety and efficiency of the hydraulic conveying part in the deep sea mining system. Figure 14 shows the profiles of particles' total kinetic energy in the statistical domain at different observation sections for the three different swirling pipes and no blade pipe, and the total kinetic energy $\left(E_{k t}\right)$ of particle group is averaged over time.

In no blade pipe, $E_{k t}$ gradually increases at different observation planes along the flow direction and then tends to be stable. In the swirling pipes, $E_{k t}$ first increases rapidly, then decreases and gradually stabilizes. It can also be seen from Fig. 14 that $E_{k t}$ becomes significantly larger at the place where the helical blades are installed, $E_{k t}$ in BL1 is more than 4 times that of the NBL, and $E_{k t}$ in BL2 is about 2 times that of the NBL. And in the subsequent downstream region, $E_{k t}$ of BL1 and BL2 is still larger than that of NBL, which is also the reason why the swirling pipe has a larger pressure drop than the no blade pipe, especially at the helical blade region $(4 \mathrm{D}<y \leqslant 12 \mathrm{D})$.

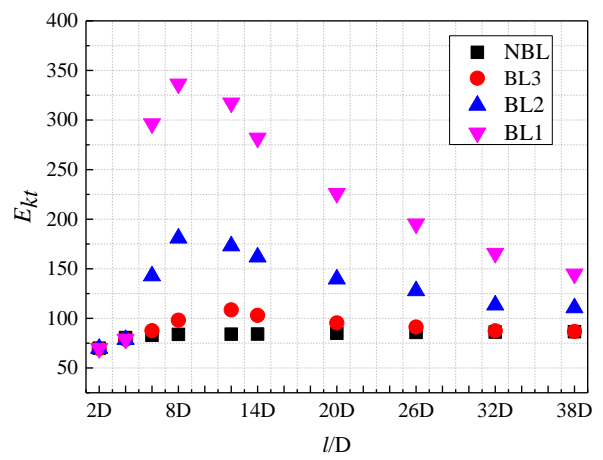

Fig. 14. Total kinetic energy of particle group at different observation planes for the three different swirling pipes and no blade pipe.

\section{Conclusion}

To improve the conveying efficiency and safety of hydraulic lifting system, a new type of pipeline conveying system with helical blade is proposed in this study. By using the CFD-DEM coupling method, the liquid-solid two-phase flow characteristics are analyzed for the swirling pipes 
and no blade pipe, and the following important conclusions are summarized.

(1) The distribution of axial velocity exhibits a symmetrical warhead-like structure for the cases of no blade pipe, while the distinct asymmetric distributions are presented in the swirling pipes. The tangential velocities of the swirling pipes are clearly larger than that of the cases of no blade pipe. The swirling number decreases as the increasing of helix angle of helical blade within swirling pipe.

(2) The fluid vorticity of the inlet region in front of the blade is low, and the large vorticity magnitude appears in the helical blade region, and then gradually decreases along the flow direction. As the decreasing of helix angle of helical blade pipes, the vorticity magnitude increases sequentially, and the vortex core structure of the flow field was gradually enriched.

(3) The total pressure decreases for all cases along the flow direction, and eventually they're going to be equal at the outlet of all cases. However, the total pressures for the swirling pipes decrease rapidly after the fluid enters the helical blades region, reflecting the difference energy efficiency of the swirling pipes.

(4) Along the flow direction, the local concentration decreases at different observation planes for the cases of no blade pipe and tends to stabilize, while the local concentration first increases and then decreases in the swirling pipes.

(5) The drag force and kinetic energy of particles in no blade pipe gradually decrease and tends to be stable along the flow direction, however, the drag force and kinetic energy of particles in the swirling pipes first increase and then decrease, and gradually stabilized. The drag force and kinetic energy of particles for the cases of the swirling pipes clearly larger that of no blade pipe in the entire observation area.

\section{ACKNOWLEDGEMENTS}

The author wishes to acknowledge support given to him by Natural Science Foundation of Jiangsu Province of China (No.BK20191459, BK20180978) and Postgraduate Research \&Practice Innovation Program of Jiangsu Province of China (SJCX19_0610).

\section{REFERENCES}

Algifri, A. H., R. Bhardwaj and Y. V. N. Rao (1987). Prediction of the decay process in turbulent swirl flow. Proceedings of the Institution of Mechanical Engineers. Part C: Journal of Mechanical Engineering Science 201,279-283.

Ariyaratne, C. and T. F. Jones (2007). Design and optimization of swirl pipe geometry for particle-laden liquids. AIChE Journal 53(4), 757-768.

Asakura, K., T. Asari and I. Nakajima (1997). Simulation of solid-liquid flows in a vertical pipe by a collision model. Powder Technology 94, 201-206.

Bai, X. N., S. G. Hu, D. F. Zhang and H. B. Qin (2001). Advances and applications of solidliquid two-phase flow in pipeline hydrotransport. Journal of Hydrodynamics 16 , 303-311.

Baker, E. (2007). Quantifying the global distribution of hydrothermal venting. The 2 nd Seabed Scientific Forum-Continental Margin and Ocean Ridge, Key Laboratory of Submarine Geosciences, The Second Institute of Oceanography, SOA.

Bali, T. and B. A. Sarac (2014). Experimental investigation of decaying swirl flow through a circular pipe for binary combination of vortex generators. International Communications in Heat and Mass Transfer 53, 174-179.

Cao, B., J. X. Xia, P. F. Hei and Y. J. Bu (2012). Assessment on motion state changes and critical condition of coarse particles in pipeline hydraulic transport. Journal of Sediment Research 90, 319-325.

Derksen, J. J. (2005). Simulations of confined turbulent vortex flow. Computers \& Fluids 34, 301-318.

Durand, R. (1952). The hydraulic transportation of coal and solid materials in pipes. Collage of National Coal Board, London, 39-52.

Ergun, S. (1952). Fluid flow through packed columns. Chemical Engineering Progress 48 , 89-94.

Escudier, M., J. Bornstein and N. Zehnder (1980). Observations and LDA measurements of confined turbulent vortex flow. Journal of Fluid Mechanics 98, 49.

Fokeer, S., I. S. Lowndes and D. M. Hargreaves (2010). Numerical modelling of swirl flow induced by a three-lobed helical pipe. Chemical Engineering and Processing 49, 536-546.

Fokeer, S., I. S. Lowndes and S. Kingman (2009). An experimental investigation of pneumatic swirl flow induced by a three lobed helical pipe. International Journal of Heat and Fluid Flow 30, 369-379.

Ghaya, H., R. Guizani, H. Mhiri and P. Bournot (2019). CFD study of the effect of geometrical shape of separation blades on the rotor performance of an annular centrifugal extractor (ACE). Journal of Applied Fluid Mechanics 12(4), 1189-1202.

Gillies, R. G. and C. A. Shook (1994). Concentration distribution of sand slurries in horizontal pipe flow. Particulate Science and 
Technology 12(1), 45-69.

Hein, J. (2003). Cobalt-rich ferromanganese crusts: global distribution, composition, origin and research activities. International Seabed Authority 5, 36-89.

Karimi, H. and A. M. Dehkordi (2015). Prediction of equilibrium mixing state in binary particle spouted beds: Effects of solids density and diameter differences, gas velocity, and bed aspect ratio. Advanced Powder Technology 26(5), 1371-1382.

Klančišar, M., T. Schloen, M. Hriberšek and N. Samec (2016). Analysis of the effect of the swirl flow intensity on combustion characteristics in liquid fuel powered confined swirling flames. Journal of Applied Fluid Mechanics 9(5), 2359-2367.

Li, H. and Y. Tomita (1994). Characteristics of swirling flow in a circular pipe. Journal of Fluids Engineering 116, 370-373.

Li, H. and Y. Tomita (1996). An experimental study of swirling flow pneumatic conveying system in a horizontal pipeline. Journal of Fluids Engineering 118,526-530.

Li, H. and Y. Tomita (2000). Particle velocity and concentration characteristics in a horizontal dilute swirling flow pneumatic conveying. Powder Technology 107, 144-152.

Newitt, D. M. and J. F. Richardson (1955). Hydraulic conveying of solids. Nature 75, 800-801.

Prabhansu, S. Rajmistry, S. Ganguli, P. Chandra, M. Kr karmakar and P. Kr Chatterjee (2017). Numerical analysis of Gas-Solid behavior in a cyclone separator for circulating fluidized bed system. Journal of Applied Fluid Mechanics 10(4), 1167-1176.

Schlichting, H. (1979). Boundary Layer Theory. ISBN 0-07-055334-3, 7th Edition.

Shih, T., W. W. Liou, A. Shabbir, Z. Yang and J. Zhu (1995). A new k-€ eddy viscosity model for high reynolds number turbulent flows. Computers \& Fluids 24, 227-238.

Tang, D. S., N. Yang and X. Jin (2013). Hydraulic lifting technique with vertical pipe for deepsea coarse mineral particles. Mining and Metallurgical Engineering 33(5), 1-8.

Tsuji, T., K. Yabumoto and T. Tanaka (2008). Spontaneous structures in three-dimensional bubbling gas-fluidized bed by parallel DEMCFD coupling simulation. Powder Technology 184,132-140.

Wen, C. Y. and Y. H. Yu (1955). Mechanics of fluidization. Chemical Engineering Progress, Symposium Series 62,100-111.

Xia, J. X., J. R. Ni and C. Mendoza (2004). Hydraulic lifting of manganese nodules through a riser. Journal of Offshore Mechanics and Arctic Engineering 126, 7277.

Xiao, Y. X., L. Yang, L. Cao and Z. Wang (2014). Distribution of marine mineral resource and advances of deep-sea lifting pump technology. Journal of Drainage and Irrigation Machinery Engineering 32, 319326.

Xiong, C. Q., M. Y. Zhang and Q. Y. Fan (2012). Numerical simulation of external flow field around low drag car and its error analysis. Automotive Engineering 34(1), 36-45.

Xu, Z. L. (1998) A new model for interpretating the velocity distribution of a heterogeneous flow in horizontal pipe. Hydraulic Coal Mining \& Pipeline Transportation 04, 34-40.

Zhou, F. B., S. Y. Hu, Y. K. Liu, C. Liu and T. Q. Xia (2014). CFD-DEM simulation of the pneumatic conveying of fine particles through a horizontal slit. Particuology 16, 196-205.

Zhou, J., C. Du, S. Liu and Y. Liu (2016). Comparison of three types of swirling generators in coarse particle pneumatic conveying using CFD-DEM simulation. Powder Technology 301, 1309-1320. 